The Astrophysical Journal, 681:470-481, 2008 July 1

(C) 2008. The American Astronomical Society. All rights reserved. Printed in U.S.A.

\title{
THE EFFECT OF TURBULENT INTERMITTENCY ON THE DEFLAGRATION TO DETONATION TRANSITION IN SUPERNOVA Ia EXPLOSIONS
}

\author{
Liubin Pan, J. Craig Wheeler, and John Scalo \\ Astronomy Department, University of Texas at Austin; panlb@astro.as.utexas.edu \\ Received 2007 October 26; accepted 2008 March 11
}

\begin{abstract}
We examine the effects of turbulent intermittency on the deflagration to detonation transition (DDT) in Type Ia supernovae. The Zel'dovich mechanism for DDT requires the formation of a nearly isothermal region of mixed ash and fuel that is larger than a critical size. We primarily consider the hypothesis by Khokhlov et al. and Niemeyer and Woosley that the nearly isothermal, mixed region is produced when the flame makes the transition to the distributed regime. We use two models for the distribution of the turbulent velocity fluctuations to estimate the probability as a function of the density in the exploding white dwarf that a given region of critical size is in the distributed regime due to strong local turbulent stretching of the flame structure. We also estimate lower limits on the number of such regions as a function of density. We find that the distributed regime, and hence perhaps DDT, occurs in a local region of critical size at a density at least a factor of 2-3 larger than predicted for mean conditions that neglect intermittency. This factor makes the transition density much larger than the empirical value from observations in most situations. We also consider the intermittency effect on the more stringent conditions for DDT by Lisewski et al. and Woosley. We find that a turbulent velocity of $10^{8} \mathrm{~cm} \mathrm{~s}^{-1}$ in a region of size $10^{6} \mathrm{~cm}$, as required by Lisewski et al., is rare. We expect that intermittency has a weaker effect on the Woosley model with a stronger DDT criterion. The predicted transition density from this criterion remains below $10^{7} \mathrm{~g} \mathrm{~cm}^{-3}$ after accounting for intermittency using our intermittency models.
\end{abstract}

Subject headings: stars: interiors — supernovae: general — turbulence

\section{INTRODUCTION}

A successful model for Type Ia supernova (SN Ia) explosions is required to produce a deflagration to detonation transition (DDT) by observational constraints. A pure deflagration model results in a lower exploding kinetic than observed (Khokhlov 1991; Gamezo et al. 2003; Röpke \& Hillebrandt 2005), and pure detonation leads to the overproduction of iron group elements and the underproduction of intermediate elements (Branch et al. $1982,1983)$. The density $\rho_{\text {tr }}$ at which the transition occurs determines the amount of the nickel produced (Höflich 1995; Höflich \& Khokhlov 1996; Dominguez et al. 2001). Therefore a prediction of $\rho_{\mathrm{tr}}$, consistent with the observed nickel production, is essential to a DDT theory for SNe Ia.

The mechanism by which the DDT occurs still remains a mystery. The most studied candidate is the Zel'dovich mechanism, which requires the existence of an almost isothermal region of mixed ash and fuel that is larger than a critical size $l_{c}$ to drive a supersonic shock that is sufficiently strong to sweep over the entire star (Khokhlov et al. 1997, hereafter KOW97; Niemeyer \& Woosley 1997, hereafter NW97). One hypothesis is that a nearly isothermal region is produced by turbulent preconditioning. KOW97 argued that to produce an almost isothermal mixture of ash and fuel, the laminar flame must be quenched by turbulent stretching, at least locally. This might allow the cold fuel to mix with the ash both thermally by electron conduction and chemically by diffusivity without being burned. They assumed that the criterion for quenching a flame is that the turbulent velocity at the laminar flame thickness must be larger than the laminar flame speed. NW97 gave a similar argument based on the distributed flame burning regime in turbulent combustion. The criterion for a distributed flame is expressed in terms of the Gibson scale at which the turbulence velocity equals the laminar flame speed. If the Gibson scale is smaller than the laminar flame thickness, turbulent stretching can generate structures within the flame and the flame is in the distributed regime. NW97 speculated that in this regime flames can be temporally quenched in some regions, which can host the detonation after being homogenized in temperature and composition by turbulent mixing. The criterion for the distributed regime is equivalent to that for flame quenching used by KOW97. Both criteria give the same condition on the turbulence intensity for given laminar flame properties (see $\S 2$ ). As the density in the star drops due to the overall expansion, it is easier for turbulence to affect the laminar flame because of the decrease in the flame speed and the increase in flame thickness. With presumed turbulence parameters, the criterion for the turbulence intensity, determined by the robustness of laminar flames disturbed by turbulent motions, translates to a transition density $\rho_{\text {tr }}$ for the DDT.

Several uncertainties exist in the simple model given by these two early studies. First, it is not clear whether the criterion used by KOW97, equivalent to that for a distributed regime (NW97), is sufficient for flame breaking. How, or even if, flames are quenched is still an open question. Second, it is uncertain whether (local) flame quenching is indeed necessary to produce a nearly isothermal region. Finally, later studies by Lisewski et al. (2000a; see also Lisewski et al. 2000b) and Woosley (2007) find that entering the distributed regime, while probably a necessary condition, is not sufficient to cause the DDT to occur. Based on a requirement for turbulent transport to be efficient at producing a shallow temperature and composition gradient around the laminar flame, Lisewski et al. (2000a) find that the turbulent velocity at the scale $10^{6} \mathrm{~cm}$ needed for a detonation is very large, $\sim 10^{8} \mathrm{~cm} \mathrm{~s}^{-1}$. Woosley (2007) claims that the DDT occurs only when the turbulent flame thickness exceeds a critical length scale. We show in $\S 2$ that the two criteria, although arising from different physical considerations, are basically equivalent. The corresponding condition is more stringent than that assumed by KOW97 and NW97. 
In this paper we examine the effect of turbulent intermittency on the onset of distributed burning that may relate to the DDT. Despite the uncertainties listed above, we will mainly consider the model by KOW97 and NW97 and use it to illustrate the potential importance of intermittency in SN Ia explosions. Our calculations can be applied to the criteria by Lisewski et al. (2000a) and Woosley (2007) in a straightforward way. A quantitative analysis using their criteria requires data for laminar flame properties and critical length scales at densities below $10^{7} \mathrm{~g} \mathrm{~cm}^{-3}$ that are not immediately available (see $\S 2$ ). We give a qualitative discussion of the intermittency effect on their DDT models.

Intermittency is an important concept in turbulence theory. It is characterized by intense local events, e.g., strong stretching at small scales, which occur at a frequency much larger than predicted from a Gaussian distribution (see, e.g., Frisch 1995). The physical origin of intermittency in turbulent flows is the spatial inhomogeneity in the energy dissipation rate: most kinetic energy is viscously dissipated in the finest structures, e.g., vortex tubes, which occupy only a small volume fraction. These rare but intense dissipative structures give rise to a spatially inhomogeneous and intermittent distribution for the turbulent intensity and the stretching rate. Intermittency is shown as broad exponential tails in the probability distribution for the stretching rate or the dissipation rate at small scales (see $\S 3$ ). The tails get broader at smaller scales, meaning that the probability of finding an extreme turbulent stretching rate or intensity increases with decreasing scales.

According to one-dimensional simulation results by KOW97 and NW97, the critical size, $l_{c}$, of the isothermal region required for a DDT via the Zel'dovich mechanism is much smaller, especially at large densities, than the expected integral length scale for the bouyancy-driven turbulence in SN Ia explosions. This suggests that only a small flame region with a sufficiently strong local turbulence intensity may be needed to trigger a detonation. Turbulent intermittency, which indicates the existence of regions of small sizes where the turbulent stretching is much larger than the average value over the flow, is therefore expected to have important consequences for DDT. The transition could happen earlier at a higher transition density $\rho_{\text {tr }}$ than predicted by models using the average turbulent intensity. At higher densities, much larger turbulent intensity is required for the DDT, but the rapid decrease of $l_{c}$ with increasing density makes an earlier DDT possible for two reasons. First, the probability is larger to find regions of smaller sizes $l_{c}$ with extreme turbulent stretching rate or intensity. Second, there are more regions of smaller size available as candidates to host the detonation. Clearly, the intermittency effect accounts for the intuitive dependence of $\rho_{\text {tr }}$ on $l_{c}$; the smaller the critical size, the easier it may be for the transition to happen. To what degree the intermittency effect increases $\rho_{\text {tr }}$ is the main question we investigate in this paper.

In $\S 2$, we review the criteria for the DDT in models by KOW97, NW97, Lisewski et al. (2000a), and Woosley (2007) and formulate a new criterion taking into account the effect of intermittency. We describe two intermittency models by Oboukhov (1962) and Kolmogorov (1962) and by She \& Leveque (1994) in $\S 3$. Using the intermittency models, we evaluate the transition density from the new criteria in $\S 4$. Our results are summarized and discussed in $\S 5$.

\section{CRITERIA FOR THE DDT}

The criterion used in NW97 to judge whether a flame is in the distributed regime, which was also assumed to be the condition for the DDT, is to compare the Gibson scale $l_{\mathrm{G}}$ with the laminar flame thickness $l_{f}$. The Gibson scale is defined such that $\delta u\left(l_{\mathrm{G}}\right)=S_{l}$ where $\delta u(l)$ is the amplitude of the velocity fluctuations at the scale $l$ [or equivalently the velocity difference over a scale $l$, i.e., $\delta u(l)=u(l+x)-u(x)]$ and $S_{l}$ is the laminar flame speed. ${ }^{1}$ If $l_{\mathrm{G}} \gtrsim l_{f}$, the turbulence cannot internally disturb the flame and the turbulence in effect wrinkles the flame. This is called the flamelet regime. Only when $l_{\mathrm{G}} \lesssim l_{f}$ can turbulence stretch the flame efficiently to generate structures within the flame and the turbulent combustion enter the distributed regime. The condition $l_{\mathrm{G}} \lesssim l_{f}$ is equivalent to $\delta u\left(l_{f}\right) \gtrsim \delta u\left(l_{\mathrm{G}}\right)=S_{l}$ since $\delta u(l)$ is an increasing function of the scale $l{ }^{2}$ The latter, which means that the turbulent velocity fluctuation $\delta u\left(l_{f}\right)$ at the scale of the flame thickness $l_{f}$ is larger than the laminar flame speed, is the criterion used in KOW97 for flame quenching and the DDT.

Following KOW97, we introduce a factor of $K \sim 1$ in the criterion to account for the uncertainty in the flame breaking mechanism, i.e., $\delta u\left(l_{f}\right) \geq K S_{l}$. We will consider two values for $K$, i.e., $K=1$ and $K=8$ (KOW97; For $K=8$, to quench a flame, the Gibson scale has to be $K^{3}=512$ times smaller than the flame width). This criterion can also be written in terms of timescales. Noting that the turbulent stretching timescale, $\tau_{t}$, at the flame thickness is $\tau_{t}\left(l_{f}\right)=l_{f} / \delta u\left(l_{f}\right)$ and that the nuclear reaction timescale, $\tau_{n}$, is related to the flame speed $\tau_{n}=l_{f} / S_{l}$, the criterion is equivalent to $\tau_{t}\left(l_{f}\right)<\tau_{n} / K$, i.e., to break the flame the stretching timescale at the flame thickness must be smaller than the nuclear burning timescale (see Niemeyer \& Kerstein 1997).

To apply this criterion, the Kolmogorov (1941) scaling $\delta u(l)=$ $\bar{\epsilon}^{-1 / 3} l^{1 / 3}$ is usually used to calculate $\delta u\left(l_{f}\right)$ from the turbulent velocity fluctuations at large scales where $\bar{\epsilon}$ is the average dissipation rate in the flow. From this scaling, the criterion can be written as (KOW97, NW97)

$$
\bar{\epsilon}^{1 / 3} l_{f}^{1 / 3}>K S_{l}
$$

or

$$
\bar{\epsilon}>K^{3} S_{l}^{3} / l_{f}=K^{3} \epsilon_{f}
$$

where $\epsilon_{f}$ is defined as $S_{l}^{3} / l_{f}$. Although we use the convenient criterion (2) in terms of the dissipation rate in our calculations, the turbulent stretching is more fundamental and we will use the concept of the flame stretching in our discussions.

The laminar flame speed and thickness depend on the chemical composition and the density (Timmes \& Woosley 1992, KOW97). In Table 1, we list the flame speed and the flame thickness as a function of density for a white dwarf with half carbon and half oxygen, mainly taken from Timmes \& Woosley (1992). The laminar speed decreases and the thickness increases quickly with decreasing density $\rho$; therefore $\epsilon_{f}$ decreases rapidly with decreasing $\rho$ as shown in Table 1 . The average dissipation rate is estimated to be $\bar{\epsilon}=U^{3} / L$ where $U$ and $L$ are the characteristic velocity and length scales of the turbulence, normally set

\footnotetext{
1 Note that NW97, accounting for the cellular stabilization effect against instabilities, e.g., the Landau-Darrieus instability, defined $l_{G}$ as the scale where the turbulent velocity exceeds the effective cellular flame speed. This does not introduce a significant difference in the estimate of $l_{G}$ since the effective cellular speed is close to the laminar speed and has a very weak dependence on scale; see their Fig. 1.

2 This is also equivalent to the diffusivity criterion by Niemeyer \& Kerstein (1997) for the onset of distributed regime and flame extinction at a Prandtl number larger than unity, which is the case for white dwarfs. Their criterion was motivated by the observation that, at a Prandtl number different from unity, two previous criteria proposed for the flamelet breakdown and for the flame quenching, using the ratio of the flow viscous length scale to the flame thickness and the ratio of the viscous timescale to the reaction timescale, respectively, are not equivalent.
} 
TABLE 1

The Laminar Flame Speed, the Flame Thickness, and the Critical Length for a White Dwarf with Half Carbon and Half Oxygen

\begin{tabular}{|c|c|c|c|c|}
\hline $\begin{array}{c}\rho \\
\left(10^{9} \mathrm{~g} \mathrm{~cm}^{-3}\right)\end{array}$ & $\begin{array}{c}S_{l} \\
\left(10^{5} \mathrm{~cm} \mathrm{~s}^{-1}\right)\end{array}$ & $\begin{array}{c}l_{f} \\
(\mathrm{~cm})\end{array}$ & $\begin{array}{c}\epsilon_{f} \\
\left(10^{15} \mathrm{~cm}^{2} \mathrm{~s}^{-3}\right)\end{array}$ & $\begin{array}{c}l_{c} \\
(\mathrm{~cm})\end{array}$ \\
\hline $2 \ldots$ & 75.8 & $9.35(-5)$ & $4.66(9)$ & $7(1)$ \\
\hline $0.5 \ldots$ & 18.1 & $9.46(-4)$ & $6.27(6)$ & \\
\hline $0.1 \ldots \ldots+\ldots$ & 2.33 & $2.75(-2)$ & $4.60(2)$ & $2(2)$ \\
\hline $0.05 \ldots \ldots \ldots \ldots \ldots \ldots$ & 0.599 & $5.19(-1)$ & 0.414 & $1.3(3)^{\mathrm{a}}$ \\
\hline $0.03 \ldots$ & $0.26^{\mathrm{b}}$ & $1.78^{\mathrm{b}}$ & $0.98(-2)$ & $5(3)$ \\
\hline $0.01 \ldots \ldots \ldots \ldots \ldots \ldots \ldots \ldots$ & $4.72(-2)$ & 4.22 & $2.59(-5)$ & $2(5)$ \\
\hline
\end{tabular}

Notes. - The values of $S_{l}$ and $l_{f}$ are mainly taken from Table 3 of Timmes \& Woosley 1992. The value of $l_{c}$ is mainly taken from NW97. We also include numbers (marked) from KOW97 because their results are very similar to NW97 despite the different details in the two models. Numbers in parentheses are powers of 10 .

${ }^{\text {a }}$ Read from Fig. 6 in KOW97.

b Read from Fig. 7 in KOW97.

by motions on the large, driving scale. At large scales, the turbulence is driven by the Rayleigh-Taylor instability. The length scale $L$ might be expected to be about the size, $R_{f}$, of the flame region, $L \simeq R_{f} \sim 10^{8} \mathrm{~cm}$ and the velocity scale to be about the Rayleigh-Taylor velocity at this scale $U \sim 0.5\left(g_{\text {eff }} L\right)^{1 / 2} \simeq$ $10^{8} \mathrm{~cm} \mathrm{~s}^{-1}$, where the effective gravity is taken to be $\sim g_{\text {eff }}=$ $5 \times 10^{8} \mathrm{~cm} \mathrm{~s}^{-2}$ (KOW97, NW97). Khokhlov (1995), however, showed that motions at scales larger than $10^{6} \mathrm{~cm}$ freeze out due to the overall expansion of the star. In that case, $L \sim 10^{6}-10^{7} \mathrm{~cm}$ and $U \sim 10^{7} \mathrm{~cm} \mathrm{~s}^{-1}$. We will take $U$ and $L$ as parameters. Note that the criterion of equation (2) depends on $U$ and $L$ through the dissipation rate. Given the dissipation rate $\bar{\epsilon}$, the critical density below which the inequality (eq. [2]) is satisfied can be obtained using $\epsilon_{f}$ as a function of $\rho$ in Table 1. For example, if $U \sim 100 \mathrm{~km} \mathrm{~s}^{-1}$ and $L \sim 100 \mathrm{~km}, \bar{\epsilon} \sim 10^{14} \mathrm{~cm}^{2} \mathrm{~s}^{-3}$ and we find that, from interpolation in Table $1, \bar{\epsilon}$ is larger than $\epsilon_{f}$ at a density less than $\sim 4 \times 10^{7} \mathrm{~g} \mathrm{~cm}^{-3}$. Therefore criterion (2) predicts a transition density $\rho_{\text {tr }} \simeq 4 \times 10^{7} \mathrm{~g} \mathrm{~cm}^{-3}$ for $K=1$ (see KOW97 and NW97). If $K=8$, the predicted transition density is smaller, $\rho_{\text {tr }} \sim 1.5 \times 10^{7} \mathrm{~g} \mathrm{~cm}^{-3}$. In the second line of Table 2, we give the predicted $\rho_{\text {tr }}$ for different values of the parameters, which decreases with decreasing dissipation rate $\bar{\epsilon}$. The numbers in parentheses correspond to $K=8$.

When using the criterion of equation (2), we need to keep in mind that the spatial fluctuations of $\epsilon$ (see $\S 3$ ) are completely neglected and the criterion only applies to the overall situation in the combustion flow. We will refer to this criterion as the mean criterion. When the mean criterion is met, the only implication is that the combustion is in the distributed regime in general. Considering the intermittency of turbulence, i.e., the spatially inhomogeneous distribution of the stretching strength, there can be places where the stretching rate is much weaker than the average. These places could still be in the flamelet stage while most other places are in the distributed regime. Or conversely, even if the mean criterion (2) is not satisfied, one cannot exclude the possibility of a region existing that experiences strong stretching and gets into the distributed regime when most of the structure is still in the flamelet regime. This latter fact is important for the deflagration to detonation transition. The fact that the DDT does not require the entire star to be in the distributed regime but instead only needs a region that is much smaller than the white dwarf radius (see below, KOW97), coupled with the intrinsic intermittency, suggests that DDT could occur earlier than predicted by equation (2) and hence at a larger transition density. The
TABLE 2

Predicted Transition Densities $\rho_{\mathrm{TR}}$ For Various Models

\begin{tabular}{cccccc}
\hline \hline Models & $\mathrm{A}^{\mathrm{a}}$ & $\mathrm{B}^{\mathrm{a}}$ & $\mathrm{C}^{\mathrm{a}}$ & $\mathrm{D}^{\mathrm{a}}$ & $\mathrm{E}^{\mathrm{a}}$ \\
\hline Mean criterion .......... $^{2}$ & $6.9(3.3)$ & $5.5(2.3)$ & $4.1(1.5)$ & $3.0(1.0)$ & $2.0(-)$ \\
Lognormal $^{\mathrm{b}} \ldots \ldots \ldots \ldots .$. & $27(10)$ & $11(4.9)$ & $10(4.4)$ & $9.4(4.0)$ & $4.3(-)$ \\
Lognormal $^{\mathrm{c}} \ldots \ldots \ldots \ldots \ldots$. & $23(7.6)$ & $9.7(4.9)$ & $8.4(3.9)$ & $7.1(3.0)$ & $4.3(-)$ \\
Log-Poisson ............. $^{2}$ & $24(8.6)$ & $9.5(4.3)$ & $8.7(3.8)$ & $7.9(3.3)$ & $3.8(-)$ \\
\hline
\end{tabular}

Notes.-Units are $10^{7} \mathrm{~g} \mathrm{~cm}^{-3}$. The numbers in parentheses are the results predicted if the Gibson scale has to be 512 times smaller than the flame thickness for the transition to the distributed regime. In case E, (-) indicates that the transition density is smaller than $10^{7} \mathrm{~g} \mathrm{~cm}^{-3}$, which cannot be well estimated since we only have data to $10^{7} \mathrm{~g} \mathrm{~cm}^{-3}$ in Table 1 .

${ }^{a}$ A: $U=10^{8} \mathrm{~cm} \mathrm{~s}^{-1}, L=10^{8} \mathrm{~cm}, \bar{\epsilon}=10^{16} \mathrm{~cm}^{2} \mathrm{~s}^{-3}$; B: $U=10^{7} \mathrm{~cm} \mathrm{~s}^{-1}$, $L=10^{6} \mathrm{~cm}, \bar{\epsilon}=10^{15} \mathrm{~cm}^{2} \mathrm{~s}^{-3} ; \mathrm{C}: U=10^{7} \mathrm{~cm} \mathrm{~s}^{-1}, L=10^{7} \mathrm{~cm}, \bar{\epsilon}=10^{14} \mathrm{~cm}^{2} \mathrm{~s}^{-3}$; D: $U=10^{7} \mathrm{~cm} \mathrm{~s}^{-1}, L=10^{8} \mathrm{~cm}, \bar{\epsilon}=10^{13} \mathrm{~cm}^{2} \mathrm{~s}^{-3} ; \mathrm{E}: U=10^{6} \mathrm{~cm} \mathrm{~s}^{-1}$, $L=10^{6} \mathrm{~cm}, \bar{\epsilon}=10^{12} \mathrm{~cm}^{2} \mathrm{~s}^{-3}$.

b The predicted transition density assuming a perfect fit of the distribution $P\left(\epsilon_{l_{c}}\right)$ by lognormal. This is the upper limit for $\rho_{\text {tr }}$ since the lognormal approximation may break down and overestimate the distribution at the far tail.

${ }^{c}$ The lower limit for the transition density assuming the lognormal approximation applies only up to $5 \sigma$.

detonation can be triggered locally when a region appears that is larger than the critical size and enters the distributed regime due to a strong local stretching. It is important to study the degree to which this intermittency effect increases the transition density, which is constrained by observations. Clearly the answer depends on the critical size, which we consider next.

The question of how large the isothermal region with wellmixed ash and fuel has to be for a detonation was studied by KOW97 (see also NW97). In their model, the DDT occurs via the Zel'dovich mechanism (Zel'dovich et al. 1970), where the mixed region begins spontaneous ignition at the place with the minimum induction time, and the flame propagates with a phase speed equal to the inverse of the spatial gradient of the induction time, which is large for nearly isothermal and well-mixed regions and is not limited by the speed of light. As the phase speed decreases below the Chapman-Jouget speed, a shock forms just ahead of the flame front. Whether this shock can explode the whole star depends on the strength of the shock when entering the pure fuel, which is determined by the size of the isothermal region. If the isothermal region is small and the shock is weak, the flame front and shock separate with the flame front lagging behind the shock, and the shock cannot make the whole star explode. The critical strength of the shock corresponds to a critical size of the isothermal region, over which the shock can be strengthened. Using one-dimensional simulations, KOW97 and NW97 obtained the critical size, $l_{c}$, which depends on the density and the chemical composition. It is interesting to note that at early time when the density is large, the required size is much smaller than that at later times. We will show this has important consequences. The critical size is much smaller than what current numerical simulations can resolve; therefore the problem of the intermittent stretching at scale $l_{c}$ cannot be addressed by simulations.

We need a local criterion to check whether a region of a given size $l$, in particular $l_{c}$, is in the distributed regime or not. For that purpose, we use a local average dissipation rate $\epsilon_{l}$ (see eq. [8] in $\S 3$ for a definition) in a region of size $l$ to replace $\bar{\epsilon}$. Following the same argument that leads to equation (2), the criterion for a region of size $l$ being in the distributed regime is

$$
\epsilon_{l}>K^{3} \epsilon_{f}
$$


where we have used the refined similarity hypothesis by Kolmogorov (1962) (see eq. [9] in $\S 3$ ). Due to the random nature of turbulent flows, $\epsilon_{l}$ is stochastic, and a statistical approach is necessary. We therefore ask the question: what is the probability that any region of size $l$ is in the distributed regime? This is given by the cumulative probability $P\left(\epsilon_{l}>K^{3} \epsilon_{f}\right)$. To answer this question, we need the probability distribution $P\left(\epsilon_{l}\right)$ of $\epsilon_{l}$. Fortunately, this distribution has been extensively studied in the intermittency models for turbulence, which we describe in $\S 3$. Although these models were originally proposed for homogeneous and isotropic turbulence, we will assume they apply to $\mathrm{SNe}$ Ia where the turbulence is stratified and may not arrive at isotropy even at very small scales. Once the distribution is specified, one can calculate the probability of finding that a region of given size $l_{c}$ is in the distributed regime,

$$
P\left(\epsilon_{l_{c}}>K^{3} \epsilon_{f}\right)=\int_{K^{3} \epsilon_{f}}^{\infty} P\left(\epsilon_{l_{c}}\right) d \epsilon_{l_{c}},
$$

which depends on the density through $l_{c}$ and $\epsilon_{f}$. An immediate examination of equation (4) shows that, at larger density, the lower limit of the integral $K^{3} \epsilon_{f}$ is larger because of the fast flame speed and the small flame thickness. This tends to decrease the probability. However, at larger density, $l_{c}$ is smaller and the intermittency of turbulence tells us that the tail of the distribution $P\left(\epsilon_{l}\right)$ is broader for smaller $l$. This tends to counteract the decrease of the cumulative probability due to the larger lower integral limit at higher densities.

Furthermore, for smaller $l_{c}$, there are more regions of size $l_{c}$ available in the star. This could make the transition occur significantly earlier, with a transition density considerably larger than that predicted by equation (2). We need to multiply the probability that a given region of size $l_{c}$ is in the distributed regime by the number, $N_{l_{c}}$, of regions of size $l_{c}$ available in order to calculate the number of regions that are both larger than $l_{c}$ and in the distributed regime at any given density. We assume that the deflagration to detonation transition happens when

$$
N_{l_{c}} P\left(\epsilon_{l_{c}}>K^{3} \epsilon_{f}\right)=1 \text {. }
$$

Since we are concerned with the flame being stretched into the distributed regime, only locations around the flame front are of interest when calculating $N_{l_{c}}$. Therefore, we only count regions in the vicinity of the flame front. $N_{l_{c}}$ depends on the size, $R_{f}$, of the flame region and the flame geometry. A typical value for $R_{f}$ is $10^{8} \mathrm{~cm}$ (Khokhlov 1995), which could be smaller at an earlier time. We will set $R_{f} \simeq L$ in our calculations in order to decrease the number of parameters. Note that $R_{f}>L$ when the freezeout effect is considered and therefore the number $N_{l_{c}}$ we use is a lower limit. If the flame region is a two-dimensional spherical front, $N_{l_{c}} \sim 4 \pi R_{f}^{2} / l_{c}^{2}$. If the flame structure is highly convoluted, it may have a fractal dimension larger than 2. In that case, $N_{l_{c}}$ is larger. The upper limit for $N_{l_{c}}$ is $\simeq 4 \pi R_{f}^{3} / 3 l_{c}^{3}$, which applies if the flame geometry is close to three-dimensional. Again, we take the lower limit $N_{l_{c}}=4 \pi R_{f}^{2} / l_{c}^{2}$; thus the transition density we will get is a lower limit.

As discussed in $\S 1$, Lisewski et al. (2000a) and Woosley (2007) find that entering the distributed regime is not sufficient for the DDT to occur and give criteria stronger than that used in KOW97 and NW97. Lisewski et al. (2000a) considered how turbulent transport affects the temperature and composition profile around a laminar flame. They assumed that, at any point, turbulence translates the temperature and composition by a distance $l_{t}$, over which turbulence can transport during a local induction time $\tau_{i}$. The distance $l_{t}$ is a function of position since $\tau_{i}$ depends on local temperature and composition. It is estimated by the length scale of a turbulent eddy with turnover time equal to $\tau_{i}$, i.e., $l_{t} / \delta u\left(l_{t}\right)=\tau_{i}$. Using the Kolmogorov (1941) scaling, we get $l_{t}=\bar{\epsilon}^{1 / 2} \tau_{i}^{3 / 2}$. For given turbulence intensity, temperature and composition profiles around a laminar flame front can be calculated from the translation. Clearly, more efficient turbulent transport gives a shallower temperature and composition profile, which is needed for detonation. By checking whether the resulting profiles, as initial conditions to solve the one-dimensional hydrodynamic equations, can lead to a detonation, Lisewski et al. (2000a), obtained a condition for the DDT on the turbulent intensity. They found that, for a successful detonation, the turbulent velocity has to be $\sim 10^{8} \mathrm{~cm} \mathrm{~s}^{-1}$ at the scale $10^{6} \mathrm{~cm}$. This condition is stronger than just entering the distributed regime. ${ }^{3}$ Since the expected turbulent velocity at scale $10^{6} \mathrm{~cm}$ is $10^{6}-$ $10^{7} \mathrm{~cm} \mathrm{~s}^{-1}$, Lisewski et al. (2000a) concluded that a DDT via the Zel'dovich mechanism in SNe Ia is unlikely. However, considering the spatial inhomogeneity of turbulent intensity, i.e., intermittency, it is possible for regions of size $10^{6} \mathrm{~cm}$ with large enough turbulent velocity to arise.

The result of Lisewski et al. (2000a) motivated Röpke (2007) to study the probability of finding a region of size $10^{6} \mathrm{~cm}$ with a turbulent rms velocity of $\sim 10^{8} \mathrm{~cm} \mathrm{~s}^{-1}$. Using data from threedimensional numerical simulations with a turbulent subgrid-scale method, Röpke (2007) analyzed the velocity fluctuations at the grid size $\left(10^{6} \mathrm{~cm}\right)$ and obtained a fat exponential tail for large velocity fluctuations that extends up to $10^{8} \mathrm{~cm} \mathrm{~s}^{-1}$. The large velocity fluctuations seem likely to be located at the trailing edge of a bubble-like feature (Röpke 2007). This confirms the intermittency in the turbulent combustion flow in SNe Ia; there exist grid cells where the turbulent intensity is much stronger than the average. From the probability of finding a grid cell with required turbulent intensity, Röpke concluded that the DDT triggered by a local cell with large velocity fluctuations is possible but probably rare. In our notations, the probability is given by $P\left(\epsilon_{10^{6} \mathrm{~cm}}>\right.$ $10^{18} \mathrm{~cm}^{2} \mathrm{~s}^{-3}$ ), where $10^{18} \mathrm{~cm}^{2} \mathrm{~s}^{-3}$ corresponds to the dissipation rate in a region of size $10^{6} \mathrm{~cm}$ with a rms velocity of $10^{8} \mathrm{~cm} \mathrm{~s}^{-1}$. We will calculate this probability and consider the availability of such regions using two intermittency models given in $\S 3$ and compare with the results of Röpke (2007) in $\S 4$.

Woosley (2007) proposed a new criterion for the DDT based on a calculation of the distributed flame width using an eddy diffusivity approximation. Making an analogy to the estimate of the laminar flame thickness, Woosley (2007) obtained the distributed flame width $\lambda$ from the equation $\lambda \simeq\left[D(\lambda) \tau_{n}\right]^{1 / 2}$ where $D(\lambda)=$ $\delta u(\lambda) \lambda$ is the eddy diffusivity at scale $\lambda$ and $\tau_{n}$ is the nuclear reaction timescale. Using the Kolmogorov (1941) scaling for $\delta u(\lambda)$, the distributed flame width is given by $\lambda=\bar{\epsilon}^{1 / 2} \tau_{n}^{3 / 2}$ (note that this formula for $\lambda$ is similar to $l_{t}$ in Lisewski et al. 2000a). Woosley (2007) assumed that the condition for detonation is that the minimum burning timescale in the distributed flame is smaller than the sound crossing time over the distributed flame width $\lambda$, or equivalently, $\lambda \gtrsim r_{\text {sonic }}^{\min }$, where $r_{\text {sonic }}^{\min }$ is the sound crossing length over the minimum burning timescale in the distributed flame. The minimum sound crossing length is thus the critical size of

\footnotetext{
3 This condition can be converted into a form that can be directly compared with eq. (2). Roughly speaking, the physical condition for a detonation in this model is that $l_{t}$ at the laminar flame front is larger than $l_{c}$, i.e., a shallow temperature gradient can be produced over a critical size around the flame front. Requiring $l_{t}>l_{c}$, we get the criterion $\bar{\epsilon}>\left(l_{c} / l_{f}\right)^{2}\left(\tau_{n} / \tau_{i}\right)^{3} \epsilon_{f}$. Considering that $\tau_{i}$ defined by Lisewski et al. (2000a) is smaller than the nuclear timescale $\tau_{n}$ and that $l_{c} \gg l_{f}$, this condition is much stronger than the condition of eq. (2). Note that this condition is similar to the criterion of Woosley (2007) given below.
} 
the distributed flame width for detonation. The criterion $\lambda \gtrsim r_{\text {sonic }}^{\text {min }}$ is equivalent to $\bar{\epsilon} \gtrsim\left(r_{\text {sonic }}^{\min }\right)^{2} / \tau_{n}^{3}$. Noting that $\tau_{n}=l_{f} / S_{l}$, the condition can be written as $\bar{\epsilon} \gtrsim\left(r_{\text {sonic }}^{\text {min }} / l_{f}\right)^{2} \epsilon_{f}$. Since $r_{\text {sonic }}^{\text {min }}$ given in Table 4 of Woosley (2007) is close to $l_{c}$ listed in Table 1 in this paper, we will use $l_{c}$ instead of $r_{\text {sonic }}^{\min }$ for simplicity, i.e.,

$$
\bar{\epsilon} \gtrsim\left(l_{c} / l_{f}\right)^{2} \epsilon_{f}
$$

which is much stronger than equation $(2)$, because $l_{c}$ is much larger than the laminar flame thickness $l_{f}$. This condition can be used to determine the transition density $\rho_{\text {tr }}$ by the same calculation process as in the case of the criterion equation (2). Note that, except a factor of $\left(\tau_{n} / \tau_{i}\right)^{3}$, this criterion is basically equivalent to that given in footnote 3 for the requirement by Lisewski et al. (2000a). We find that, for the reasonable turbulence parameters listed in Table 2 , the criterion results in a transition density below $10^{7} \mathrm{~g} \mathrm{~cm}^{-3}$ and we cannot specify it due to the lack of data for $l_{c}, S_{l}$, and $l_{f}$ at densities below $10^{7} \mathrm{~g} \mathrm{~cm}^{-3}$.

In his estimate for $\rho_{\mathrm{tr}}$, Woosley (2007) used $U=10^{8} \mathrm{~cm} \mathrm{~s}^{-1}$ at scale $L=10^{6} \mathrm{~cm}$ throughout the calculations, based on the result by Röpke (2007) on the possibility of the existence of regions of size $10^{6} \mathrm{~cm}$ with a rms velocity of $10^{8} \mathrm{~cm} \mathrm{~s}^{-1}$. With these turbulence parameters he derived $\rho_{\mathrm{tr}}=10^{7} \mathrm{~g} \mathrm{~cm}^{-3}$. Clearly, in Woosley's calculation, the intermittency effect implicitly contributes to the transition density obtained because, as discussed earlier, a turbulent rms velocity of $10^{8} \mathrm{~cm} \mathrm{~s}^{-1}$ at $10^{6} \mathrm{~cm}$ can only arise from intermittency.

The intermittency effect for the criterion of Woosley (2007) can be included more consistently in our formulation. Instead of considering a single special scale $10^{6} \mathrm{~cm}$, our model specifies intermittency over a continuous range of scales corresponding to critical sizes at different densities. Following the same steps that lead to equation (5), we incorporate the intermittency effect in the DDT model of Woosley (2007) and obtain a criterion,

$$
N_{l_{c}} P\left[\epsilon_{l_{c}}>\left(l_{c} / l_{f}\right)^{2} \epsilon_{f}\right]=1
$$

which only differs from equation (5) by the lower limit in the cumulative probability. We will discuss about this criterion in $\S 4$.

We point out that the eddy diffusivity method used by Woosley (2007) to approximate the combined action of the turbulent advection and the microscopic diffusivity is an oversimplification. This procedure implicitly assumes a smooth structure in the distributed flame and neglects the fluctuations of temperature and concentration, which may be important in determining the effective width of distributed flames.

\section{INTERMITTENCY}

Kolmogorov's 1941 theory assumes that the energy transfer in the inertial range is equal to the average dissipation rate $\bar{\epsilon}$ in the flow and is the same throughout the inertial scales down to the viscous scale where the kinetic energy is removed. This assumption, together with the similarity hypothesis, predicts that the statistics of the velocity difference (or the velocity fluctuations) at any inertial scale are completely determined by the average dissipation rate $\bar{\epsilon}$. However, fluctuations in the dissipation rate clearly exist, as can be seen from the formula for the local viscous dissipation rate, $\epsilon(\mathbf{x}, t)=(\nu / 2) \sum_{i, j}\left(\partial_{i} u_{j}+\partial_{j} u_{i}\right)^{2}$, which is a function of the fluctuating velocity field. The spatial fluctuations in $\epsilon$ are well illustrated by the intense dissipation structures at small scales, such as vortex tubes. This effect needs to be taken into account for a more accurate prediction of the scaling behavior of the velocity difference (Landau \& Lifshitz 1944). The statistics of the velocity difference over a separation $l$ depends on the distribution of the dissipation rate over regions of size $l$, which is defined as (e.g., Kolmogorov 1962)

$$
\epsilon_{l}(\boldsymbol{x}, t)=\frac{3}{4 \pi l^{3}} \int_{\left|\boldsymbol{x}^{\prime}\right|<l} \epsilon\left(\boldsymbol{x}+\boldsymbol{x}^{\prime}, t\right) d \boldsymbol{x}^{\prime} .
$$

Clearly, the mean of $\epsilon_{l}$ is equal to $\bar{\epsilon}$ and thus is independent of $l$. This means that the average energy flux over all the inertial scales is constant. The $\epsilon_{l}$ distribution is essential to the intermittency models for turbulence. Note that this distribution is exactly what we need in our calculations for the transition of the turbulent combustion to the distributed regime by turbulent stretching and quenching described in $\S 2$, equations (4) and (5).

Intermittency in turbulence is usually expressed in terms of the scaling behavior of the structure functions $\left\langle\delta u(l)^{p}\right\rangle \sim l^{\zeta_{p}}$, where $\delta u(l)=u(x+l)-u(x)$ is the (longitudinal) velocity difference and $\zeta_{p}$ is the scaling exponent for the $p$ th-order structure function. Kolmogorov's 1941 theory predicts that the exponent $\zeta_{p}$ goes with $p$ as $\zeta_{p}=p / 3$. However, experimental data (e.g., Anselmet et al. 1984) have shown departures from this linear relation and $\zeta_{p}$ increases significantly slower than $p / 3$ at large values of $p$. This "anomalous" scaling is referred to as intermittency. The data indicate broader and broader tails for the distribution of $\delta u(l)$ at smaller and smaller scales, e.g., the kurtosis of the distribution, $\left\langle\delta u(l)^{4}\right\rangle /\left\langle\delta u(l)^{2}\right\rangle^{2} \propto l^{\zeta_{4}-2 \zeta_{2}}$, increases with decreasing $l$ because $\zeta_{4}<2 \zeta_{2}$. The distribution of $\delta u(l)$ is fatter for smaller separations $l$. The anomalous scaling is fundamentally caused by the fluctuations in the dissipation rate $\epsilon_{l}$. Applying the refined similarity argument for homogeneous and isotropic turbulence (Kolmogorov 1962), the velocity difference over a separation $l$ can be related to the dissipation rate $\epsilon_{l}$,

$$
\delta u(l) \sim \epsilon_{l}^{1 / 3} l^{1 / 3}
$$

(note that the Kolmogorov [1941] theory uses $\bar{\epsilon}$ ). The structure functions are then given by

$$
\left\langle\delta u(l)^{p}\right\rangle \propto\left\langle\epsilon_{l}^{p / 3}\right\rangle l^{p / 3} .
$$

Clearly, the departure from the linear scaling for the velocity difference comes from the statistics of the dissipation rate. Assuming $\left\langle\epsilon_{l}^{p}\right\rangle \propto l^{\tau_{p}}$ (e.g., She \& Leveque 1994), we have

$$
\zeta_{p}=p / 3+\tau_{p / 3}
$$

Developing a physical model for $\tau_{p}$ that satisfies the experimental result for $\zeta(p)$ has been the main task of intermittency theories. Although we are mainly concerned with the distribution of $\epsilon_{l}$, discussions of the structure functions are necessary because they are directly measurable in experiments and give important information and constraints on the $\epsilon_{l}$ distribution. We will use two intermittency models in our calculations: the lognormal model (Oboukhov [1962] and Kolmogorov [1962]) and the logPoisson model by She \& Leveque (1994).

\subsection{The Lognormal Model}

Oboukhov (1962) and Kolmogorov (1962) developed the first intermittency model. In this model, the distribution of $\epsilon_{l}$ is assumed to be lognormal (Kolmogorov 1962). A justification for this "natural" distribution for $\epsilon_{l}$ was given by Yaglom (1966). Imagine the cascade progress as successive eddy fragmentations 
from the integral scale $L$ to the dissipation scale $\eta$. The statistics of the energy flux at an inertial scale $l$ (or equivalently the dissipation rate $\epsilon_{l}$ ) depends on the fragmentations before the scale is reached. The total number $N$ of steps that lead to the scale $l$ is proportional to $N \sim \ln (L / l)$. Defining $\chi_{i}=\epsilon_{i} / \epsilon_{i-1}$ as the ratio of the energy transfer rates at two successive fragmentation steps, the energy flux at the scale $l$ can be expressed in the ratios (see e.g., Monin \& Yaglom 1975),

$$
\epsilon_{l}=\epsilon_{L} \chi_{1} \chi_{2} \ldots \chi_{N}
$$

where $\epsilon_{L}$ is the dissipation rate at the integral scale (or the transfer flux at the largest scale), $\epsilon_{L} \simeq \bar{\epsilon}$. Due to the randomness in the fragmentation process the ratios, $\chi_{i}$, are stochastic variables. Assuming a self-similar fragmentation process, the distributions of the ratios are similar and $\ln \left(\epsilon_{l} / \epsilon_{L}\right)=\sum_{i=1}^{N} \ln \left(\chi_{i}\right)$ is expected to be Gaussian from the central limit theorem,

$$
p\left(\epsilon_{l}\right) d \epsilon_{l}=\frac{1}{\sqrt{2 \pi \sigma_{l}^{2}}} \exp \left\{-\frac{\left[\ln \left(\epsilon_{l} / \bar{\epsilon}\right)+\sigma_{l}^{2} / 2\right]^{2}}{2 \sigma_{l}^{2}}\right\} d \ln \left(\epsilon_{l} / \bar{\epsilon}\right),
$$

where the variance $\sigma_{l}^{2}$ is proportional to the number of steps $\sigma_{l}^{2}=\mu \ln (L / l)$ with $\mu$ being a parameter to be determined by experimental data and the $\sigma_{l}^{2} / 2$ term in the numerator in the exponential is to guarantee the mean $\left\langle\epsilon_{l}\right\rangle$ is equal to the overall average dissipation rate $\bar{\epsilon}$. This distribution will be used later to calculate the probability (eq. [4]) for a region of a given size being in the distributed regime.

The scaling behavior of $\epsilon_{l}$ can be derived by integrating equation (13),

$$
\left\langle\epsilon_{l}^{p}\right\rangle \propto l^{-(1 / 2) \mu p(p-1)}
$$

which gives $\tau_{p}=-\frac{1}{2} \mu p(p-1)$. From equation (11), we have

$$
\zeta_{p}=p / 3-\frac{1}{18} \mu p(p-3)
$$

Therefore $\mu=2-\zeta_{6}$, which can be obtained from the results of experiments and simulations. It has been found that $\mu \simeq 0.2$ (Frisch 1995; Biskamp 2003). The relation (15) agrees with experiments quite well at small $p$ but starts to exhibit deviation at $p \gtrsim 10$ and gives an unrealistic maximum and turnover at $p>16$, violating the requirement that the $\zeta(p)-p$ curve must be monotonic and concave (Frisch 1995). Simulations by Wang et al. (1996) suggest that this disagreement corresponds to the departure of the distribution for $\ln \left(\epsilon_{l} / \bar{\epsilon}\right)$ from normal at scales close to the dissipation scale. They find that, at these scales, the distribution of $\ln \left(\epsilon_{l} / \bar{\epsilon}\right)$ shows a negative skewness, meaning that the lognormal distribution overestimates the probability in the very high $\epsilon_{l}$ tail. However, the distribution of $\epsilon_{l}$ agrees with lognormal very well in the inertial range away from the dissipation scale, and the agreement is better and better for larger and larger scales (Wang et al. 1996). Fortunately the critical scale we are concerned with is well within the inertial range (see $\S 4$ ), and according to Figure 6 in Wang et al. (1996) the lognormal fit is very good at least up to the $4 \sigma$ tail. They also show that the fit gets better as the Reynolds number increases. More recent simulations by Yeung et al. (2006) with resolutions up to $2048^{3}$ obtained similar results. The lognormal distribution gives a very good fit to $4 \sigma$ and only deviates by a factor of 2 at the $5 \sigma$ tail. They also find that the negative skewness gets closer to zero with increasing Reynolds number. The Reynolds number in SNe Ia is $\operatorname{Re} \sim 10^{14}$ for typical velocity scale $10^{7} \mathrm{~cm} \mathrm{~s}^{-1}$, length scale $10^{7} \mathrm{~cm}$, and viscosity $1 \mathrm{~cm}^{2} \mathrm{~s}^{-1}$. This is much larger than in any of the current simulations. Therefore one may expect that the lognormal distribution probably applies even further out on the tail for the inertial scales of the turbulence in SNe Ia. However, the departure of the predicted $\zeta(p)-p$ curve from the experiments (with high $\mathrm{Re}$ ) at $p \gtrsim 10$ suggests that, even at huge Reynolds number, the lognormal fit eventually breaks down at some large $\epsilon_{l}$ in the tail. Therefore we need to be careful when using the lognormal model. We will discuss this point further in the calculations given in $\S 4$.

Another issue is that the distribution of $\epsilon_{l}$ has a physical cutoff in a realistic system due to the finite viscosity. Since the intermittency is stronger at smaller scales, the cutoff in the distribution of $\epsilon_{l}$ is probably larger for smaller $l$ and obtains a maximum at the dissipation scale, $\eta$. For Kolmogorov scaling, the cutoff in the distribution of $\epsilon_{\eta}$ is given by $\bar{\epsilon} \mathrm{Re}^{1 / 2}$. Since $\operatorname{Re} \sim 10^{14}$ in $\mathrm{SNe} I \mathrm{Ia}$, this maximum dissipation rate is far beyond that required to break flames at density $\lesssim 10^{8} \mathrm{~g} \mathrm{~cm}^{-3}$. Therefore ignoring this maximum cutoff does not affect our result. However, the largest available dissipation rate at an inertial scale $l$ is probably smaller than the cutoff in the distribution of $\epsilon_{\eta}$ and thus may affect the calculation for the cumulative probability defined in equation (4) if the cutoff in the distribution $P\left(\epsilon_{l_{c}}\right)$ is close to or even smaller than $K^{3} \epsilon_{f}$. Since the lognormal model does not address the cutoff in the distribution of $\epsilon_{l}$, we will neglect this potential effect in this model.

On the other hand, the log-Poisson model we consider in the next section gives a maximum dissipation rate at each inertial scale, corresponding to the strongest dissipative structures at that scale. In that model, a nonzero cumulative probability in equation (5) requires the lower limit $K^{3} \epsilon_{f}$ in equation (4) to be smaller than the maximum.

\subsection{The Log-Poisson Model}

A major success in the intermittency theory is the model by She \& Leveque (1994). In this model, She and Leveque studied the hierarchy of dissipation intensity in structures of size $l$ and, by invoking an unknown "hidden symmetry," they related the characteristic dissipation rates in structures of different intensity levels to the strongest dissipative structures. This relation gives a prediction of $\tau_{p}$ as a function of $p$, which only depends on the proprieties of the most intermittent structures. Assuming that the dissipation rate in regions of size $l$ containing the most intense structures exhibits a scaling $\propto l^{-2 / 3}$ (see explanation in the Appendix) and the most intermittent structures are filamentary, corresponding to a codimension of 2 , She and Leveque obtained a $\zeta(p)-p$ relation, which is in excellent agreement with experimental data. The hidden symmetry" has been immediately interpreted as a log-Poisson process (Dubrulle 1994; She \& Waymire 1995) in a multiplicative cascade model. In this section, we adopt the log-Poisson version of the She-Leveque model. The original presentation by She \& Leveque (1994) is given in the Appendix.

In a multiplicative model, the dissipative rates at two scales $l_{2}$ and $l_{1}\left(l_{1}>l_{2}\right)$ are related by a multiplicative factor $W_{l_{1} l_{2}}$,

$$
\epsilon_{l_{2}}=W_{l_{2} l_{1}} \epsilon_{l_{1}} .
$$

The average $\left\langle W_{l_{1} l_{2}}\right\rangle$ is equal to unity since $\left\langle\epsilon_{l_{1}}\right\rangle=\left\langle\epsilon_{l_{2}}\right\rangle=\bar{\epsilon}$. She \& Waymire (1995) speculated that $W_{l_{2} l_{1}}$ consists of two events. 
First is the amplification of the dissipation rate in the cascade, which tends to produce singular structures with $\epsilon_{l_{2}} \propto\left(l_{1} / l_{2}\right)^{\gamma}$ approaching infinity as $l_{2}$ goes to 0 . The meaning of $\gamma$ is discussed below. To ensure $\left\langle W_{l_{1} l_{2}}\right\rangle=1$, a second event is required to reduce $W_{l_{1} l_{2}}$. She \& Waymire (1995) called this event the modulation defects since it modulates the singular structures. The defects were assumed to be a discrete Poisson process. Each of the defects decreases $W_{l_{1} l_{2}}$ by a factor of $\beta$, thus

$$
W_{l_{2} l_{1}}=\left(l_{1} / l_{2}\right)^{\gamma} \beta^{n}
$$

if there are $n$ defect events in the cascade. The number $n$ of the events that occur in the cascade from the scale $l_{1}$ to $l_{2}$ obeys a Poisson distribution,

$$
P(n)=\exp \left(-\lambda_{l_{1} l_{2}}\right) \frac{\lambda_{l_{1} l_{2}}^{n}}{n !},
$$

where $\lambda_{l_{1} l_{2}}$ is the mean number of the defect events in the cascade, which is expected to be proportional to the total number of the cascade steps, i.e., $\lambda_{l_{1} l_{2}} \propto \ln \left(l_{1} / l_{2}\right)$. In fact, $\lambda_{l_{1} l_{2}}$ can obtained by taking the average of equation (17) and requiring $\left\langle W_{l_{1} l_{2}}\right\rangle=$ 1. Using the identity $\sum_{n=0}^{\infty}\left(\alpha^{n} / n !\right)=\exp (\alpha)$, we get $\left\langle\beta^{n}\right\rangle=$ $\exp \left[(\beta-1) \lambda_{l_{1} l_{2}}\right]$ for the Poisson distribution of equation (18); therefore,

$$
\lambda_{l_{1} l_{2}}=\frac{\gamma \ln \left(l_{1} / l_{2}\right)}{1-\beta} .
$$

In this model, there is a largest dissipation rate at each scale. Clearly the largest dissipation rate is achieved if there is no defect, i.e., $n=0$, in a cascade from the integral scale $L$ to the scale $l$ of interest; thus the largest dissipation rate is equal to $\epsilon_{L}(L / l)^{\gamma}$, which corresponds to $\epsilon_{l}^{(\infty)}$ in the Appendix. Similarly $n=1$ gives the second strongest dissipative rate at a given scale, and so on.

From equations (16) and (17), we have

$$
\ln \left(\epsilon_{l_{2}} / \bar{\epsilon}\right)=\ln \left(\epsilon_{l_{1}} / \bar{\epsilon}\right)+\gamma \ln \left(l_{1} / l_{2}\right)+n \ln (\beta)
$$

thus, using the Poisson distribution for $n$, the distribution for the dissipation rate at $l_{2}$ can be derived from that at any scale $l_{1}$ larger than $l_{2}$. In particular, we consider deriving the distribution of $\epsilon_{l}$ at any scale $l$ from the integral scale $L$. The distribution function of $\epsilon_{L}$ at the integral scale depends on how the energy is injected in the flow; thus it is not universal and may vary from flow to flow. Therefore the function form cannot be specified. However, there is a strong constraint for its width. Since $\epsilon_{L} \simeq \bar{\epsilon}$, the distribution of $\ln \left(\epsilon_{L} / \bar{\epsilon}\right)$ is expected to be very narrow around $\ln \left(\epsilon_{L} / \bar{\epsilon}\right) \simeq 0$ and hence to be approximately a delta function. We denote the distribution of $\ln \left(\epsilon_{L} / \bar{\epsilon}\right)$ as $P_{L}\left[\ln \left(\epsilon_{L} / \bar{\epsilon}\right)\right]$. It then follows from equations (18) and (20) that

$$
\begin{aligned}
P\left(\epsilon_{l}\right) d \epsilon_{l}= & \sum_{n=0}^{\infty} \exp (-\lambda) \frac{\lambda^{n}}{n !} P_{L} \\
& \times\left[\ln \left(\epsilon_{l} / \bar{\epsilon}\right)-\gamma \ln (L / l)-n \ln (\beta)\right] d \ln \left(\epsilon_{l} / \bar{\epsilon}\right),
\end{aligned}
$$

where $\lambda=\lambda_{L l}=\gamma \ln (L / l) /(1-\beta)$. Each term in equation (21) represents the contribution from dissipation structures of dif- ferent levels, e.g., the $n=0$ term corresponds to the most intensive structures of size $l$.

To compare the model with experiments and obtain the parameters, we calculate the moments $\left\langle\epsilon_{l}^{p}\right\rangle$ from the distribution equation (21),

$$
\begin{aligned}
\left\langle\epsilon_{l}^{p}\right\rangle & \propto \sum_{n=0}^{\infty} \int \exp (-\lambda) \frac{\lambda^{n}}{n !} \exp (p x) P_{L}[x-\gamma \ln (L / l)-n \ln (\beta)] d x \\
& =(l / L)^{-\gamma p} \exp (-\lambda) \sum_{n=0}^{\infty} \frac{\left(\beta^{p} \lambda\right)^{n}}{n !} \int \exp \left(x^{\prime}\right) P_{L}\left(x^{\prime}\right) d x^{\prime} \\
& =B_{p} \exp \left[-\lambda\left(1-\beta^{p}\right)\right](l / L)^{-\gamma p} \\
& =B_{p}(l / L)^{-\gamma p+\gamma\left(1-\beta^{p}\right) /(1-\beta)}
\end{aligned}
$$

where we used a variable change $x^{\prime}=x-\gamma \ln (L / l)-n \ln (\beta)$ in the second step and the identity $\sum_{n=0}^{\infty}\left(\alpha^{n} / n !\right)=\exp (\alpha)$ in the third step. The coefficients $B_{p}=\int \exp \left(p x^{\prime}\right) P_{L}\left(x^{\prime}\right) d x^{\prime}$ and $B_{0}=$ $B_{1}=1$ from the normalization of $P_{L}$ and the requirement that $\left\langle\epsilon_{L}\right\rangle=\bar{\epsilon}$, respectively.

The result equation (22) gives $\tau_{p}=-\gamma p+\gamma\left(1-\beta^{p}\right) /(1-\beta)$, which is the same as (A8) in the Appendix, meaning that the "hidden symmetry" described in the Appendix is equivalent to a $\log$-Poisson process. The parameters $\gamma$ and $\beta$ introduced here are identical to those described in the Appendix, and thus have the physical meanings explained there, i.e., $\gamma$ can be interpreted as the exponent of the dissipation rate scaling in regions containing the most intermittent structures and $\beta$ is related to the codimension $C$ of the strongest dissipation structures, $\gamma /(1-\beta)=C$ (see the Appendix for details). As discussed in the Appendix, She and Leveque argued that $\gamma=2 / 3$ and $\beta=2 / 3$ for $C=2$ corresponding to filamentary dissipation structures in incompressible turbulence. This results in $\zeta_{p}$ as a function of $p$ that agrees with the experiments with an accuracy of $1 \%$, implying equation (21) provides a good distribution for $\epsilon_{l}$. The She-Leveque formulation has been extended to supersonic turbulence (Boldyrev et al. 2002) and MHD turbulence (Muller \& Biskamp 2000) where the dissipation structures are dissipation sheets and the current sheets, respectively. For these two-dimensional dissipation structures, the codimension $C=1$ and $\beta=1 / 3$. In next section we use the log-Poisson distribution (eq. [21]) in our calculations for the cumulative probability in equation (4). We will take $\gamma=2 / 3$ and consider both filaments $(\beta=2 / 3)$ and sheets $(\beta=1 / 3)$ as the most intermittent dissipation structures.

\section{RESULTS}

\subsection{The Lognormal Model}

We are ready to calculate the probability $P\left(\epsilon_{l_{c}}>K^{3} \epsilon_{f}\right)$ using the distributions $P\left(\epsilon_{l}\right)$ given in $\S 3$. The calculation is straightforward for the lognormal distribution equation (13),

$$
\begin{aligned}
P\left(\epsilon_{l_{c}}>K^{3} \epsilon_{f}\right) & =\int_{\ln \left(K^{3} \epsilon_{f} / \bar{\epsilon}\right)}^{\infty} \frac{1}{\sqrt{2 \pi \sigma_{l_{c}}^{2}}} \exp \left[-\frac{\left(x+\sigma_{l_{c}}^{2} / 2\right)^{2}}{2 \sigma_{l_{c}}^{2}}\right] d x \\
& =\int_{\left[\ln \left(K^{3} \epsilon_{f} / \bar{\epsilon}\right)+\sigma_{l_{c}}^{2} / 2\right] / \sigma_{l_{c}}}^{\infty} \frac{1}{\sqrt{2 \pi}} \exp \left(-x^{2} / 2\right) d x \\
& =\frac{1}{2} \operatorname{erfc}\left[\frac{\ln \left(K^{3} \epsilon_{f} / \bar{\epsilon}\right)}{\sqrt{2} \sigma_{l_{c}}}+\frac{\sigma_{l_{c}}}{2 \sqrt{2}}\right]
\end{aligned}
$$


where $\bar{\epsilon} \simeq U^{3} / L, \sigma_{l_{c}}^{2}=\mu \ln \left(L / l_{c}\right) \simeq 0.2 \ln \left(L / l_{c}\right)$ and $\operatorname{erfc}(x)$ is the complementary error function. Using the $\epsilon_{f}$ and $l_{c}$ values given in Table 1, we calculated the probability as a function of the density assuming different values for the characteristic velocity $(U)$ and length $(L)$ scales. For example, if $U=100 \mathrm{~km} \mathrm{~s}^{-1}$ and $L=100 \mathrm{~km}$, the probabilities are $0.5 \times 10^{-10}, 0.042,0.9$, and 1 at $\rho=10^{8}, 5 \times 10^{7}, 3 \times 10^{7}$, and $10^{7} \mathrm{~g} \mathrm{~cm}^{-3}$, respectively if $K=1$. It is interesting to note that, at a density $3 \times 10^{7} \mathrm{~g} \mathrm{~cm}^{-3}$, $10 \%$ of the local regions of the critical size are still in the flamelet regime, although generally the flame has reached the distributed regime according to the mean criterion equation (2). With $R_{f} \simeq$ $L=100 \mathrm{~km}$, at the four densities above from high to low, the corresponding numbers $N_{l c}=4 \pi R_{f}^{2} / l_{c}^{2}$ of regions of the critical size that cover the flame front are $3 \times 10^{10}, 7 \times 10^{8}, 5 \times 10^{7}$, and $3 \times 10^{4}$. Multiplying $P\left(\epsilon_{l_{c}}>K^{3} \epsilon_{f}\right)$ with $N_{l_{c}}$ (eq. [5]), we see that there is already one region of size $l_{c}$ in the distributed regime at a density $10^{8} \mathrm{~g} \mathrm{~cm}^{-3}$. Recalling that, according to the criterion equation (2), the DDT does not occur until the density decreases to $4 \times 10^{7} \mathrm{~g} \mathrm{~cm}^{-3}$, we find that in this case the intermittency effect may increase the transition density by more than a factor of 2 .

We point out that the cumulative probability calculated from equation (23) at density $10^{8} \mathrm{~g} \mathrm{~cm}^{-3}$ in the example above comes from a little beyond the $6 \sigma$ tail of the distribution for $\ln \left(\epsilon_{l} / \bar{\epsilon}\right)$ and we need to check whether the lognormal distribution there is a good approximation. As discussed in $\S 3.1$, numerical simulations have shown that for a scale $l$ in the inertial range, the distribution of $\epsilon_{l}$ is well approximated by lognormal up to the $5 \sigma$ tail (Yeung et al. 2006). Assuming the Kolmogorov scaling, the dissipation scale in Type Ia SNe is $\eta=L \mathrm{Re}^{-3 / 4} \simeq 10^{-3} \mathrm{~cm}$ for $L \sim 10^{7} \mathrm{~cm}$ and the Reynolds number $\operatorname{Re} \simeq 10^{14}$. The critical scale of interest here, $l_{c} \sim 10^{2}-10^{4} \mathrm{~cm}$, is well between the integral scale and the dissipation scale; thus we expect that the distribution for $\epsilon_{l_{c}}$ is close to lognormal at least up to the $\sim 5 \sigma$ tail. The question is then whether the good fit extends further. Wang et al. (1996) and Yeung et al. (2006) found that the lognormal fit is better for larger Reynolds numbers; thus it is expected that the lognormal approximation probably applies to higher on the tail than $5 \sigma$. As argued in $\S 3.1$, the lognormal approximation eventually fails somewhere in the extreme tail even at high Reynolds numbers. To know exactly how far the lognormal fit extends, numerical simulations with much higher resolution are needed. We have to be careful about the validity of the lognormal approximation in the far tail because it overestimates the probability distribution for $\epsilon_{l}$ once it breaks down, and in that case equation (23) overestimates $P\left(\epsilon_{l_{c}}>K^{3} \epsilon_{f}\right)$.

Due to the complication of the validity of the lognormal distribution at the far tail, we consider two extreme cases and give the upper and lower limits for the transition density. First, we ignore the departure from lognormal and evaluate the density at which $N_{l_{c}} P\left(\epsilon_{l_{c}}>K^{3} \epsilon_{f}\right)=1$, using equation (23) for $P\left(\epsilon_{l_{c}}>K^{3} \epsilon_{f}\right)$ and Table 1 for $\epsilon_{f}$ and $l_{c}$ with different parameters $U$ and $L$. We will denote this density as $\rho_{\mathrm{LN}}$ with the subscript $\mathrm{LN}$ standing for lognormal. Interpolation was used to obtain $\epsilon_{f}$ and $l_{c}$ values not tabulated in Table 1. If the distribution of $\epsilon_{l}$ is exactly lognormal, as given by equation (13), then $\rho_{\mathrm{LN}}$ is the predicted transition density for the DDT with the intermittency taken into account. On the other hand, if the lognormal distribution overestimates the probability at the high tail, equation (23) overestimates the cumulative probability and $\rho_{\mathrm{LN}}$ is the upper limit for $\rho_{\mathrm{tr}}$. We give $\rho_{\mathrm{LN}}$ for different parameters $U$ and $L$ in the second line of Table 2 .

On the other extreme, we assume that the lognormal distribution fails to fit the distribution of $\epsilon_{l}$ beyond the $5 \sigma$ tail. This gives a lower limit for the transition density since numerical simulations have shown that the lognormal fit applies at least to $5 \sigma$. In this case, we keep track of the integral limit in the second line of equation (23) at $\rho_{\mathrm{LN}}$, which tells us which part of the tail of the distribution gives the main contribution to $P\left(\epsilon_{l_{c}}>K^{3} \epsilon_{f}\right)$ at that density. If the integral limit is smaller than five, the contribution to the cumulative probability is from within $5 \sigma$ and vice versa. We calculate the density at which the integral limit is equal to five and denote this density $\rho_{5 \sigma}$. Since the integral limit is a decreasing function of the density, if $\rho_{\mathrm{LN}}<\rho_{5 \sigma}$, the contribution to $P\left(\epsilon_{l_{c}}>K^{3} \epsilon_{f}\right)$ at density $\rho_{\mathrm{LN}}$ is from within $5-\sigma$. In this case, the cumulative probability calculated from equation (23) is valid and $\rho_{\mathrm{LN}}$ is a good estimate for the transition density. Otherwise, if $\rho_{\mathrm{LN}}>\rho_{5 \sigma}$, the contribution to the probability is from beyond the $5 \sigma$ tail, equation (23) overestimates it, and thus $\rho_{\mathrm{LN}}$ overestimates the transition density $\rho_{\text {tr }}$. In this case, $\rho_{5 \sigma}$ gives a lower limit for the transition density because at $\rho_{5 \sigma}$, we have $N_{l_{c}} P\left(\epsilon_{l_{c}}>K^{3} \epsilon_{f}\right) \gg 1$ using equation (23), which applies for $\rho \leq \rho_{5 \sigma}$. Therefore if the lognormal fit fails just beyond $5 \sigma$, we have a lower limit for the transition density, $\min \left(\rho_{\mathrm{LN}}, \rho_{5 \sigma}\right)$. We give this lower limit in the third line of Table 2.

Similar calculations can be done for the $K=8$ case. The results of the upper and lower limits for the transition density in the $K=8$ case are given in parentheses in Table 2. Comparing these results with predictions from the mean criterion (2) (the first line in Table 2), the lognormal model predicts that the intermittency effect increases the transition density by a factor of 2-3 for all the cases we list in Table 2.

We evaluate the probability of the existence of a region of size $10^{6} \mathrm{~cm}$ with a rms velocity $10^{8} \mathrm{~cm} \mathrm{~s}^{-1}$, required for the DDT by Lisewski et al. (2000a), and compare this to the numerical results of Röpke (2007). Using the lognormal distribution for $\epsilon_{l}$, we find that the requirement $P\left(\epsilon_{10^{6} \mathrm{~cm}}>10^{18} \mathrm{~cm}^{2} \mathrm{~s}^{-3}\right)$ requires conditions from the extreme tail of the distribution. The likelihood is completely negligible $\left(\sim 10^{-40}\right)$ if the velocity $U$ at the integral length scale is less than $\sim 10^{7} \mathrm{~cm} \mathrm{~s}^{-1}$. Only if $U$ is larger than $5 \times 10^{7} \mathrm{~cm} \mathrm{~s}^{-1}$ is the probability appreciably larger so that the required region might be available. For example, if $U=5 \times 10^{7} \mathrm{~cm} \mathrm{~s}^{-1}$ at $L=$ $10^{7} \mathrm{~cm}$, the probability is $\sim 10^{-11}$. This is still too small to guarantee the existence of a region as required by Lisewski et al. (2000a). The number of available candidate regions around the flame front that are $10^{6} \mathrm{~cm}$ is probably smaller than $10^{5}-10^{7}$, assuming the flame front radius is $\sim 10^{8}-10^{9} \mathrm{~cm}$. This result agrees with the conclusion of Röpke (2007) that the existence of a region as required by Lisewski et al. (2000a) is rare. To ensure such a region, the velocity at the integral scale has to be larger than $10^{8} \mathrm{~cm} \mathrm{~s}^{-1}$, which is probably impossible as discussed in $\S 2$.

We also carry out a calculation for $\rho_{\text {tr }}$ based on the criterion of Woosley (2007) taking into account the effect of intermittency. Using the lognormal distribution to calculate the cumulative probability in equation (7), we find that no regions of critical size that meet Woosley's criterion appear at density above $10^{7} \mathrm{~g} \mathrm{~cm}^{-3}$. We cannot give an exact predicted transition density for this model because we do not have data at densities below $10^{7} \mathrm{~g} \mathrm{~cm}^{-3}$ for relevant quantities listed in Table 1 . Note that Woosley (2007) obtained a transition density around $10^{7} \mathrm{~g} \mathrm{~cm}^{-3}$ under the assumption that a region that is $10^{6} \mathrm{~cm}$ with a rms velocity of $10^{8} \mathrm{~cm} \mathrm{~s}^{-1}$ is available. From our estimate above and the result in Röpke (2007), the probability that such a region exists is small; therefore it is appropriate to take the transition density predicted in Woosley (2007) as an upper limit for his DDT criterion. 


\subsection{The Log-Poisson Model}

We next consider the log-Poisson model. Using the distribution equation (21), we have

$$
\begin{aligned}
P\left(\epsilon_{l_{c}}>K^{3} \epsilon_{f}\right)= & \exp \left(-\lambda_{c}\right) \sum_{n=0}^{\infty} \frac{\lambda_{c}^{n}}{n !} \int_{\ln \left(K^{3} \epsilon_{f} / \bar{\epsilon}\right)}^{\infty} \\
& \times P_{L}\left[x-\gamma \ln \left(L / l_{c}\right)-n \ln (\beta)\right] d x \\
= & \exp \left(-\lambda_{c}\right) \sum_{n=0}^{\infty} \frac{\lambda_{c}^{n}}{n !} \\
& \times \int_{\ln \left(K^{3} \epsilon_{f} / \bar{\epsilon}\right)-\gamma \ln \left(L / l_{c}\right)-n \ln (\beta)}^{\infty} P_{L}(x) d x \\
= & \exp \left(-\lambda_{c}\right) \sum_{n=0}^{\infty} \frac{\lambda_{c}^{n}}{n !} F_{n},
\end{aligned}
$$

where $\lambda_{c}=\gamma \ln \left(L / l_{c}\right) /(1-\beta)$ and the integrals in the second line are denoted as $F_{n}$ for convenience. Note that the integral lower limit $\ln \left(K^{3} \epsilon_{f} / \bar{\epsilon}\right)-\gamma \ln \left(L / l_{c}\right)-n \ln (\beta)$ increases with $n$ because $\beta<1$; therefore $F_{n}$ is a decreasing function of $n$. An exact calculation for the cumulative probability is impossible because of the unspecified function $P_{L}$. We will neglect all the $n \geq 1$ terms and only keep the $n=0$ term in our calculation, i.e., we only include the contribution of the most intensive structures at scale $l_{c}$. Obviously, this approximation gives a lower limit for the probability and the transition density we obtain will also be a lower limit. We will show that the criterion for DDT obtained from this approximation is exact if $P_{L}$ is a delta function.

The contribution from $n=0$ is $\exp \left(-\lambda_{c}\right)=\left(l_{c} / L\right)^{\gamma /(1-\beta)} F_{0}$. For $\gamma=2 / 3$ and $\beta=2 / 3$, it is equal to $\left(l_{c} / L\right)^{2} F_{0}$ and $N_{l_{c}} P\left(\epsilon_{l_{c}}>\right.$ $\left.K^{3} \epsilon_{f}\right) \geq 4 \pi\left(R_{f} / l_{c}\right)^{2}\left(l_{c} / L\right)^{2} F_{0}=4 \pi\left(R_{f} / L\right)^{2} F_{0}$. Since the size of the flame region $R_{f} \geq L$, it means that the number of regions which are larger than the critical size and in the distributed regime is $\simeq 4 \pi F_{0}$. Since the distribution $P_{L}(x)$ is probably strongly concentrated at $x=0$, the sufficient and almost necessary condition for $F_{0} \simeq 1$ is that the integral limit $\ln \left(K^{3} \epsilon_{f} / \bar{\epsilon}\right)-\gamma \ln \left(L / l_{c}\right) \leq$ 0 , or equivalently,

$$
\bar{\epsilon}>\left(l_{c} / L\right)^{2 / 3} K^{3} \epsilon_{f}
$$

which is a convenient criterion for the DDT in the log-Poisson model. Note that this criterion is much weaker than the mean criterion equation (2). Once the condition is satisfied, at least one region of critical size that covers the flame enters the distributed regime due to the most intense stretching strength available at scale $l_{c}$.

As mentioned in $\S 3.2$, if the dissipation structures are twodimensional, $\beta=1 / 3$. In that case, the contribution from the $n=$ 0 term is $\left(l_{c} / L\right) F_{0}$ and $N_{l_{c}} P\left(\epsilon_{l_{c}}>K^{3} \epsilon_{f}\right) \geq 4 \pi\left(R_{f} / l_{c}\right)\left(R_{f} / L\right) F_{0}$, which is much larger than one if $F_{0} \gtrsim 1$. Therefore, the criterion equation (25) is a sufficient condition for the case with sheetlike dissipation structures, such as in MHD turbulence or highly compressible turbulence.

We have neglected the $n>1$ terms in equation (24), the contribution of which depends on how rapidly $P_{L}(x)$ decreases with $x>0$. We consider the extreme example where $P_{L}$ is a delta function. In this case, before the condition equation (25) is met, $F_{n}=0$ for any $n$; thus the cumulative probability is zero. When the condition is just satisfied as the density decreases, only the $n=0$ term contributes and all the $n>1$ terms are still zero, i.e., the most intensive $(n=0)$ structures at $l_{c}$ can stretch a local flame into the distributed regime while all the less intensive structures $(n \geq 1)$ still cannot. From the calculation above, we see that in this case once the $n=0$ term contributes, at least one region around the flame front experiences the largest stretching rate and enters the distributed regime. Therefore, if $P_{L}$ is a delta function, equation (25) is both the necessary and the sufficient condition. This is true for both $\beta=2 / 3$ and $\beta=1 / 3$. If $P_{L}$ is not a delta function, the tail of $P_{L}$ gives rise to the possibility that the distributed regime can emerge in a local region of critical size before the condition equation (25) is met. This could lead to an even weaker condition than equation (25). Since we expect that $\epsilon_{L}$ can only vary within a factor of a few, the condition can be weaker only by a factor of a few. Because the right-hand side of condition (25), especially $\epsilon_{f}$, depends on the density very sensitively, this would not increase the predicted $\rho_{\text {tr }}$ considerably.

Equation (25) can be easily applied to calculate the transition density using the $\epsilon_{f}$ and $l_{c}$ values in Table 1 . For example, we get $\rho_{\text {tr }}=8.7 \times 10^{7} \mathrm{~g} \mathrm{~cm}^{-3}$ for $U=100 \mathrm{~km} \mathrm{~s}^{-1}$ and $L=100 \mathrm{~km}$ if $K=1$. This result is consistent with that from the lognormal model and is also about a factor of 2 larger than the prediction by the mean criterion (2).

The transition density predicted by the log-Poisson model with different parameters for turbulence is given in the fourth line of Table 2. Again the numbers in parentheses are for $K=8$. The results are consistent with those from the lognormal model and are at least 2-3 times larger than from criterion (2).

Again we consider the possibility that there exists a region of size $10^{6} \mathrm{~cm}$ with a rms turbulent velocity of $10^{8} \mathrm{~cm} \mathrm{~s}^{-1}$ required for DDT by Lisewski et al. (2000a). The probability $P\left(\epsilon_{10^{6} \mathrm{~cm}}>10^{18} \mathrm{~cm}^{2} \mathrm{~s}^{-3}\right)$ depends on $P_{L}$, the probability distribution of the dissipation rate at the integral scale $L$. Since $P_{L}$ is probably not universal and is flow dependent, the log-Poisson model cannot give an exact estimate for the probability. Here we assume that $P_{L}$ is a delta function and see under what condition it is possible to find a required region. We find that the necessary and sufficient condition to have such a region is that $U>$ $10^{22 / 3}(L / \mathrm{cm})^{1 / 9} \mathrm{~cm} \mathrm{~s}^{-1}$. For $L \simeq 10^{7} \mathrm{~cm}, U$ has to be larger than $10^{8} \mathrm{~cm} \mathrm{~s}^{-1}$. This can be understood from the fact that, in the logPoisson model, the available kinetic energy in the most intermittent structures for dissipation is assumed to be the kinetic energy at the integral scale (see the Appendix). Since $U>10^{8} \mathrm{~cm} \mathrm{~s}^{-1}$ is probably not achievable, it is rare that a region like that required by Lisewski et al. (2000a) exists, again in agreement with Röpke (2007).

Using the same calculation that leads to equation (25), we obtain a criterion for the DDT model by Woosley (2007) accounting for the intermittency effect,

$$
\bar{\epsilon}>\left(l_{c} / L\right)^{2 / 3}\left(l_{c} / l_{f}\right)^{2} \epsilon_{f} .
$$

This is weaker than the corresponding mean criterion (6) by a factor of $\left(l_{c} / L\right)^{2 / 3}$, meaning that intermittency increases the transition density. In comparison with equation (25), the condition is stronger and thus gives a smaller transition density than that for the KOW97 and NW97 model with intermittency included. At smaller density, the critical length $l_{c}$ is larger and the factor $\left(l_{c} / L\right)^{2 / 3}$, representing the intermittency effect, is closer to unity. This implies that intermittency has a weaker effect on the transition density for the Woosley (2007) criterion than for KOW97 and NW97. Using Table 1, we again find that the condition (26) is not satisfied at densities above $10^{7} \mathrm{~g} \mathrm{~cm}^{-3}$ for the five cases listed in Table 2, i.e., the predicted transition density is still below $10^{7} \mathrm{~g} \mathrm{~cm}^{-3}$ after including intermittency (see discussion in $\S 4.1$ ).

In summary, intermittency can considerably enhance the onset of the distributed flame regime and hence increase the transition 
density in the DDT model of KOW97 and NW97. Both the intermittency models we consider here predict a transition density 2-3 times larger than from the criterion using the mean dissipation rate. This factor of 2-3 brings the transition density to be in disagreement with the observational constraints for turbulent velocity larger than $U=10^{6} \mathrm{~cm} \mathrm{~s}^{-1}$ in the case $K=1$. We discuss the implications of this result in the next section. We also find that the existence of regions of size $10^{6} \mathrm{~cm}$ with a velocity of $10^{8} \mathrm{~cm} \mathrm{~s}^{-1}$ is rare, which agrees with the numerical result of Röpke (2007). The strong DDT criterion given by Woosley (2007) gives a transition density below $\rho_{\text {tr }}=10^{7} \mathrm{~g} \mathrm{~cm}^{-3}$ even when intermittency is included. We expect that the intermittency effect is weaker for stronger DDT criteria.

\section{CONCLUSION AND DISCUSSION}

We have studied the effect of intermittency on the transition from the flamelet regime to the distributed regime in Type Ia $\mathrm{SNe}$, and hence on the transition density for the DDT model by KOW97 and NW97. In their model, the detonation occurs via the Zel'dovich mechanism that requires a nearly isothermal region larger than a critical size to drive a sufficiently strong supersonic shock. KOW97 and NW97 assumed that the almost isothermal mixture of fuel and ash can be produced once turbulence is strong enough to get the flame into the distributed regime. The DDT is assumed by KOW97 and NW97 to occur when the average flow gets into the distributed regime. We argue that the sufficient condition for the DDT is that there is one region that is larger than the critical size and in the distributed regime.

The intermittency in turbulence, as a result of the spatial inhomogeneity of the dissipation rate, gives rise to regions with strong local turbulent strength that can force the flame into the distributed regime earlier than elsewhere. Therefore the transition from the flamelet regime to the distributed regime is not spatially smooth, but intermittent. At early time when the density in the white dwarf is large, the flame has a large speed and a small width and thus resists being efficiently stretched and broken by the turbulence. At the same time, the critical size is very small. This has two effects that tend to make an early DDT likely. First, the intermittency of turbulence tells us that the probability of finding extremely strong stretching within a smaller critical size is larger. Second, there are more regions of smaller sizes available. Therefore it is possible that the DDT is triggered at a small "spot" when the density is larger than needed for the average flow to enter the distributed regime. As we pointed out in $\S 1$, the critical size as a function of the density plays an important role in determining the transition density for the DDT in our calculations.

We used two analytical intermittency models to statistically investigate when the first region appears that is both larger than the critical size and in the distributed region. This is assumed to be the time when the DDT occurs by KOW97 and NW97. We found that, for various parameters for the intensity and length scales, DDT occurs at a transition density at least $2-3$ times larger than the density at which the average flow enters the distributed regime. The transition density has been determined empirically by invoking it as a free parameter in spherically symmetric models and then computing models that best match the observed multicolor light curve shapes and magnitudes (Höflich \& Khokhlov 1996). Recognizing that the spherical models are oversimplified, they do give some guidance to the empirical constraints on the density at which DDT occurs. Höflich (1995) used this procedure to fit observations of the Branch core normal SN 1994D and preferred a value of the transition density of $2 \times 10^{7} \mathrm{gm} \mathrm{cm}^{-3}$.
Höflich et al. (1995) explored a range of transition densities in the context of pulsating delayed detonation models and favored densities in the range $(0.8-2.2) \times 10^{7} \mathrm{gm} \mathrm{cm}^{-3}$. Dominguez et al. (2001) adopted $2.3 \times 10^{7} \mathrm{gm} \mathrm{cm}^{-3}$. Allowing for an uncertainty of a factor of 2, the predicted transition densities by the mean criterion are consistent with $2 \times 10^{7} \mathrm{gm} \mathrm{cm}^{-3}$ as favored by the observations in all the cases except that with $\bar{\epsilon}=10^{16} \mathrm{~cm}^{2} \mathrm{~s}^{-3}$ and $K=1$ (Table 2). With the intermittency effects we have examined here, the transition density would be a factor of 2-3 higher. If $K=1$, all the predicted $\rho_{\text {tr }}$ are larger than $2 \times 10^{7} \mathrm{gm} \mathrm{cm}^{-3}$ by at least a factor of 2, except for the case with $U=10^{6} \mathrm{~cm} \mathrm{~s}^{-1}$. The predicted transition density with $K=8$ is $2-3$ times smaller than from $K=1$. From Table 2, the predicted $\rho_{\text {tr }}$ for the intermittency models with $K=8$ agree with the observations within a factor of 2, except the case with a large velocity scale $U=$ $10^{8} \mathrm{~cm} \mathrm{~s}^{-1}$ at the integral length scale. To avoid discrepancy with the observations, our result indicates several possibilities.

1. The large-scale motions caused by Rayleigh-Taylor instability freeze out due to the overall expansion of the star (Khokhlov 1995). The freezeout effect has to be efficient enough for the developed part of the flow to have a velocity scale of $\lesssim 10^{6} \mathrm{~cm} \mathrm{~s}^{-1}$ (see Table 2).

2. The flame is very robust. To break the flame, the local Gibson scale has to be at least $K^{3}=512$ times smaller than the flame thickness. In this case, the predicted transition density is 2-3 times smaller than that from $K=1$.

3. There is not enough time for the buoyancy-driven turbulence to fully develop down to the critical size before the predicted density for the DDT by our intermittency models is attained; thus motions at scales below the critical size are either absent or nonintermittent.

4. The DDT does not occur immediately after a region of the critical size enters the distributed regime. It may take some time for turbulence to help mix the region and make it nearly isothermal. However, the timescale for turbulence to mix a region of the critical size in the distributed regime is very small, $\lesssim 10^{-2} \mathrm{~s}$ at densities larger than $3 \times 10^{7} \mathrm{~g} \mathrm{~cm}^{-3}$. It is unlikely that the density drops much in such a short timescale.

5. Having a large enough region entering the distributed regime is not a sufficient condition for detonation. As mentioned in $\S 1$, there are several uncertainties in the simple model by KOW97 and NW97 that assumes that flame quenching, entering the distributed regime, and the DDT all occur simultaneously.

Our results allude to the possibility that the criterion by KOW97 and NW97 is too weak for the DDT, supporting the claim of Lisewski et al. (2000a) and Woosley (2007) that just entering the distributed regime is not sufficient for the DDT. We have shown that their criteria for the DDT are much stronger than just entering the distributed regime. We also studied the intermittency effect on their conditions for the DDT. We find that the existence of a region of size $10^{6} \mathrm{~cm}$ with a rms turbulent velocity of $10^{8} \mathrm{~cm} \mathrm{~s}^{-1}$, as required by Lisewski et al. (2000a) for a DDT, is rare, which is consistent with the numerical results of Röpke (2007). We have also examined the intermittency effect on the transition density for the DDT criterion by Woosley (2007). We find that the effect is weaker for the stronger criterion and does not increase $\rho_{\text {tr }}$ to above $10^{7} \mathrm{~g} \mathrm{~cm}^{-3}$. Woosley (2007) obtained $\rho_{\text {tr }}$ around $10^{7} \mathrm{~g} \mathrm{~cm}^{-3}$ because of the assumption of a strong turbulence velocity of $10^{8} \mathrm{~cm} \mathrm{~s}^{-1}$ in a region that is $10^{6} \mathrm{~cm}$. Since the existence of such a region is rare, Woosley (2007) may considerably overestimate the transition density. This may imply that the condition for DDT by Lisewski et al. (2000a) and Woosley (2007) is too strong and 
predicts a transition density smaller than that empirically determined from observations.

We have only studied the DDT in white dwarfs with an initial chemical composition of half carbon and half oxygen. In a white dwarf with more carbon, the nuclear timescale is smaller; thus, a stronger turbulent intensity is needed to break the flame. This results in a smaller transition density using the mean criterion (NW97). On the other hand, the critical size for detonation in such a white dwarf is smaller; therefore the intermittency effect could be more efficient in increasing the transition density. We were not able to perform a calculation for a chemical composition with a carbon abundance larger than 0.5 due to the lack of sufficient data for the critical length scale in this case.
We point out that the intermittency models we used were originally proposed for homogeneous and isotropic turbulence. Turbulence in SNe Ia is stratified and may not achieve homogeneity and isotropy at very small scales even if the turbulence is developed at these scales. The effect of the departure from homogeneity and isotropy on the predicted transition density is out of the scope of this paper.

We thank Elaine Oran for useful discussions. This research was supported in part by NSF grant AST-0707769 (L. P., J. C. W.) and by NASA Astrophysics Theory Program grant NAG513280 (J. S.).

\section{APPENDIX}

\section{THE SHE-LEVEQUE MODEL}

In their original paper, She \& Leveque (1994) start with the moments of the distribution $P\left(\epsilon_{l}\right)$ and use the ratios of two successive moments, $\epsilon_{l}^{(p)}=\left\langle\epsilon_{l}^{p+1}\right\rangle /\left\langle\epsilon_{l}^{p}\right\rangle \propto l^{\tau_{p+1}-\tau_{p}}$, to characterize a hierarchy of dissipative structures. This ratio can be written as $\epsilon_{l}^{(p)}=\int \epsilon_{l} Q_{p}\left(\epsilon_{l}\right) d \epsilon_{l}$, where $Q_{p}\left(\epsilon_{l}\right)=\epsilon_{l}^{p} P\left(\epsilon_{l}\right) / \int \epsilon_{l}^{p} P\left(\epsilon_{l}\right) d \epsilon_{l}$. For a typical distribution $P\left(\epsilon_{l}\right)$ that decreases monotonically and faster than any power law at large $\epsilon_{l}, Q_{p}\left(\epsilon_{l}\right)$ strongly peaks around $\epsilon_{l}^{(p)}$ for large values of $p$. Clearly $\epsilon_{l}^{(p)}$ increases with $p$, and $\epsilon_{l}^{(\infty)}=$ $\lim _{n \rightarrow \infty}\left\langle\epsilon_{l}^{p+1}\right\rangle /\left\langle\epsilon_{l}^{p}\right\rangle$ corresponds to the most intense dissipative structures at scale $l$. These strongest dissipative structures are the origin of the anomalous scaling and the scaling of $\epsilon_{l}^{(\infty)}$ with $l$,

$$
\epsilon_{l}^{(\infty)} \sim l^{-\gamma}
$$

is of fundamental importance. To determine the parameter $\gamma$, we can dimensionally write $\epsilon_{l}^{(\infty)}$ as an energy scale divided by a timescale $t_{l}$. She and Leveque argued that for the most intermittent structures this energy scale is the largest available kinetic energy (which is $\sim v_{\mathrm{rms}}^{2}$, independent of $l$ ) and assumed that $t_{l}$ exhibits a regular Kolmogorov scaling $t_{l} \sim l^{2 / 3}$; therefore $\gamma=2 / 3$. From equation (A 1 ), we have $\tau_{p+1}=\tau_{p}-\gamma$ for $p \rightarrow \infty$, or

$$
\tau_{p}=-\gamma p+C, \quad p \rightarrow \infty
$$

where the constant $C$ has a physical interpretation as the codimension of the most intermittent structures. Equation (A1) means that the dissipation rate in a region of size $l$ that encloses the most intensive structures scales with $l$ as $\epsilon_{l} \propto l^{-\gamma}$. When calculating $\left\langle\epsilon_{l}^{p}\right\rangle$ at $p \rightarrow \infty$, we need to consider the possibility of a point finding itself within a distance $l$ to the most intermittent structures, which is proportional to $l^{(D-d)}$, where $D$ is the dimension of the system and $d$ is the dimension of the most intensive structures (Frisch 1995). As $p \rightarrow \infty$, the contribution to $\left\langle\epsilon_{l}^{p}\right\rangle$ is dominated by the most intermittent structures, therefore $\left\langle\epsilon_{l}^{p}\right\rangle \propto l^{-\gamma p+D-d}$. Comparing with equation (A2), we find that $C$ corresponds to the codimension of the most intermittent structures, $C=D-d$.

In order to determine the entire hierarchy of the dissipative structures, She and Leveque argued that the intensity $\epsilon_{l}^{(p+1)}$ of the dissipative structures at level $p+1$ depends only on their immediate precursor, the structures of level $p$, from which the level $p+1$ structures directly develop, and on the most intensive structures, where the structures of all orders tend to end up. Based on this argument, they made an assumption about the hierarchy of the dissipation rates,

$$
\epsilon_{l}^{(p+1)}=A_{p}\left(\epsilon_{l}^{(p)}\right)^{\beta}\left(\epsilon_{l}^{(\infty)}\right)^{1-\beta}
$$

where the coefficients $A_{p}$ are independent of $l$ but may be flow dependent and nonuniversal. The parameter $\beta$ will be completely fixed by $\gamma$ and the codimension $C$. According to She and Leveque, this relation corresponds to a mysterious symmetry of the Navier-Stokes equation, termed "the hidden symmetry."

To derive $\tau_{p}$ from equation (A3), it is convenient to define a new variable,

$$
\pi_{l}=\epsilon_{l} / \epsilon_{l}^{(\infty)}
$$

which was introduced by Dubrulle (1994). Clearly $\epsilon_{l}^{(p)} / \epsilon_{l}^{(\infty)}=\left\langle\pi_{l}^{p+1}\right\rangle /\left\langle\pi_{l}^{p}\right\rangle$ from the definition of $\epsilon_{l}^{(p)}$. Then the "hidden symmetry" assumed by She and Leveque becomes

$$
\frac{\left\langle\pi_{l}^{p+2}\right\rangle}{\left\langle\pi_{l}^{p+1}\right\rangle}=A_{p}\left(\frac{\left\langle\pi_{l}^{p+1}\right\rangle}{\left\langle\pi_{l}^{p}\right\rangle}\right)^{\beta} .
$$


This recursion relation is solved by

$$
\frac{\left\langle\pi_{l}^{p+1}\right\rangle}{\left\langle\pi_{l}^{p}\right\rangle}=C_{p}\left\langle\pi_{l}\right\rangle^{\beta^{p}},
$$

where $C_{0}=1$ and $C_{p}=\prod_{n=0}^{p} A_{n}^{\beta n}$ for $p>0$. Equation (A6) gives

$$
\left\langle\pi_{l}^{p}\right\rangle=B_{p}\left\langle\pi_{l}\right\rangle^{\left(1-\beta^{p}\right) / 1-\beta},
$$

where $B_{0}=B_{1}=1$ and $B_{p}=\prod_{n=1}^{p-1} C_{n}$ for $p>1$. Noting that $\left\langle\pi_{l}\right\rangle=\bar{\epsilon} / \epsilon_{l}^{(\infty)} \propto\left(\epsilon_{l}^{(\infty)}\right)^{-1}$ and $\left\langle\pi_{l}^{p}\right\rangle=\left\langle\epsilon_{l}^{p}\right\rangle\left(\epsilon_{l}^{(\infty)}\right)^{-p}$, equation (A7) gives $\left\langle\epsilon_{l}^{p}\right\rangle \propto\left(\epsilon_{l}^{(\infty)}\right)^{p-\left(1-\beta^{p}\right) /(1-\beta)}$. Using equation (A1), we have

$$
\tau_{p}=-\gamma p+\gamma \frac{\left(1-\beta^{p}\right)}{1-\beta} .
$$

The parameter $\beta$ is determined by the asymptotic behavior of $\tau_{p}$ at the $p \rightarrow \infty$, equation (A2). Letting $p \rightarrow \infty$ in equation (A8) and comparing with equation (A2), we find that $\gamma /(1-\beta)=C=D-d$. Since the most intermittent structures in three-dimensional incompressible turbulence are filamentary, we have $d=1$ and $C=2$; thus $\beta=1-\gamma / 2=2 / 3$ for $\gamma=2 / 3$. Finally we arrive at the celebrated She-Leveque formulae,

$$
\tau_{p}=-2 p / 3+2\left[1-(2 / 3)^{p}\right]
$$

and

$$
\zeta_{p}=p / 9+2\left[1-(2 / 3)^{p / 3}\right]
$$

which agrees with the experimental result with an accuracy of $1 \%$. Note that this result is consistent with Kolmogorov's exact result for the third order structure function, i.e., $\zeta_{3}=1$. If the most intense structures are two-dimensional, e.g., the dissipation sheets in compressible flows (Boldyrev et al. 2002) or the current sheets in MHD (Muller \& Biskamp 2000), $\beta=1 / 3$.

\section{REFERENCES}

Anselmet, F., Gagne, Y., Hopfinger, E. J., \& Antonia, R. A. 1984, J. Fluid Mech., 140, 63

Boldyrev, S., Nordlund, A., \& Padoan, P. 2002, Phys. Rev. Lett., 89, 031102

Branch, D., Buta, R., Falk, S. W., McCall, M. L., Uomoto, A., Wheeler, J. C., Wills, B. J., \& Sutherland, P. G. 1982, ApJ, 252, L61

Branch, D., Lacy, C. H., McCall, M. L., Sutherland, P. G., Uomoto, A., Wheeler, J. C., \& Wills, B. 1983, ApJ, 270, 123

Dominguez, I., Höflich, P., \& Straniero, O. 2001, ApJ, 557, 279

Dubrulle, B. 1994, Phys. Rev. Lett., 73, 959

Frisch, U. 1995, Turbulence (Cambridge: Cambridge Univ. Press)

Gamezo, V. N., Khokhlov, A. M., Oran, E. S., Chtchelkanova, A. Y., \& Rosenberg, R. O. 2003, Science, 299, 77

Höflich, P. 1995, ApJ, 443, 89

Höflich, P., \& Khokhlov, A. M. 1996, ApJ, 457, 500

Höflich, P., Khokhlov, A. M., \& Wheeler, J. C. 1995, ApJ, 444, 831

Khokhlov, A. M. 1991, A\&A, 245, 114 1995, ApJ, 449, 695

Khokhlov, A. M., Oran, E. S., \& Wheeler, J. C. 1997, ApJ, 478, 678 (KOW97)

Kolmogorov, A. 1941, Dokl. Akad. Nauk SSR, 30, 301

1962, J. Fluid Mech., 13, 82

Landau, L. D., \& Lifshitz, E. M. 1944, Mechanics of Continuous Media (Moscow: Gostechnicisdat)
Lisewski, A. M., Hillebrandt, W \& Woosley, S. E. 2000a, ApJ, 538, 831 Lisewski, A. M., Hillebrandt, W., Woosley, S. E., Niemeyer, J. C., \& Kerstein, A. R. 2000b, ApJ, 537, 405

Monin, A. S., \& Yaglom, A. M. 1975, Statistical Fluid Mechanics (Cambridge: MIT Press)

Muller, W-C., \& Biskamp, D 2000, Phys. Rev. Lett., 84, 475

Niemeyer, J. C., \& Kerstein, A. R. 1997, NewA, 2, 239

Niemeyer, J. C., \& Woosley, S. E. 1997, ApJ, 475, 740 (NW97)

Oboukhov, A. M. 1962, J. Fluid Mech., 13, 77

Röpke, F. K. 2007, ApJ, 668, 1103

Röpke, F. K., \& Hillebrandt, W. 2005, A\&A, 431, 635

She, Z.-S., \& Leveque, E. 1994, Phys. Rev. Lett, 72, 336

She, Z.-S., \& Waymire, E. C. 1995, Phys. Rev. Lett, 74, 262

Timmes, F. X., \& Woosley, S. E. 1992, ApJ, 396, 649

Wang, L.-P., Chen, S., Brasseur, J. G., \& Wyngaard, J. C. 1996, J. Fluid Mech., 309,113

Woosley, S. E. 2007, ApJ, 668, 1109

Yaglom, A. M. 1966, Dokl. Akad. Nauk SSSR, 166, 49

Yeung, P. K., Pope, S. B., Lamorgese, A. G., \& Donzis, D. A. 2006, Phys. Fluids, 18, 065103

Zel'dovich, Y. B., Librovich, V. B., Makhviladze, G. M., \& Sivashinsky, G. I. 1970, Acta Astron., 15, 313 